\title{
Acute Care Physiotherapy Management of COVID-19 Patients in Qatar: Best Practice Recommendations
}

\author{
MS Ajimsha, PT, PhD, DO, $M B A^{1,2}$; Neeraj Gampawar, MPT ; Praveen J Surendran, MPT; Prasobh Jacob, $P T^{l}$; Reshma \\ Praveen, MPT ${ }^{l}$; Vasileios Karpouzis, MPT ${ }^{l}$;Mohamed Haneef, $P T^{l}$; Muhamed Aleef, $P T^{l}$; Shameem Ali, MPT ${ }^{l}$; Eladel \\ Bouguerra, $P T^{l}$; Noora Almudahka PT, $M B A^{l}$ \\ ${ }^{1}$ Department of Physiotherapy, Hamad Medical Corporation, Doha, Qatar \\ ${ }^{2}$ Corresponding author: Email: ajimshaw.ms@gmail.com;Ph;0097455021106
}

\begin{abstract}
This document outlines best practice recommendations for an acute care physiotherapy for patients with COVID-19, with an emphasis on critical care rehabilitation including the patients in extra corporeal membrane Oxygenation (ECMO) support, developed for practice in Qatar but adaptable within any settings. This recommendation is the result of a combination of systematic evidence search, subsequent critical evaluation of retrieved evidence and consensus process. The agreed recommendations were integrated into a physiotherapy clinical reasoning algorithm. It includes recommendations on physiotherapy referral, screening, management categories and best practice recommendations. It is intended for use by physiotherapists and other relevant stakeholders in the acute care setting caring for adult patients with suspected and/or confirmed COVID-19.
\end{abstract}

Keywords: Coronavirus; COVID-19; SARS-Cov-2; infection; Physiotherapy; rehabilitation.

\section{Introduction}

Severe acute respiratory syndrome coronavirus 2 (SARS-CoV-2) is a new virus that emerged in 2019 and causes Coronavirus Disease 2019 (COVID-19) (del Rio and Malani PN, 2019; WHO, 2020(a)). The disease was first identified in December 2019 in Wuhan (China), and has spread globally, resulting in the ongoing pandemic (Hui DS et al., 2020; WHO, 2020(b)). SARS-CoV-2 is highly contagious. Individuals with COVID-19 can present with an influenza-like illness and respiratory tract infection whose primary symptoms are fever (89\%), cough $(68 \%)$, fatigue $(38 \%)$, sputum production $(34 \%)$ and/or shortness of breath (19\%) (Guan W et al., 2020). It has been reported that it takes about 7.0 days (4.0-8.0) from onset of symptoms to hospitalization, about 8.0 days (5.0-13.0) to develop shortness of breath, and 10.5 days (7.0-14.0) to receive mechanical ventilation mainly because of acute respiratory distress syndrome (ARDS). Current reports estimate that $80 \%$ of cases are asymptomatic or mild; $15 \%$ of cases are severe (infection requiring oxygen), and $5 \%$ are critical requiring mechanical ventilation and life support (WHO, 2020 (a)).

COVID-19 patients who develop pneumonia often require invasive mechanical ventilation and care in the intensive care unit (ICU) (Zhou F et al., 2020). Being a novel disease, the short term and long-term consequences of the COVID-19 are anticipated based on prior knowledge gained from general critical care population especially those suffering with ARDS. The ill-effects of mechanical ventilation, sedation, paralysis, immobilization and prolonged bed rest have already been well established (Hodgson et al., 2016). In addition to reduced muscle strength and decreased physical function, it also leads to impaired respiratory and swallowing function (Chen J et al., 2020). These patients are also known to suffer from cognitive dysfunction and ICU delirium (Parry SM et al., 2015). Besides the above-mentioned short-term effects, the long-term effects include reduced exercise capacity, 
dependence on activities of daily living and reduced quality of life. (Dijkstra-Kersten SMA et al., 2020; Mikkelsen ME et al., 2009).

The standard method of diagnosis is by real-time reverse transcription-polymerase chain reaction (rRT-PCR) from a nasopharyngeal swab (NIH, 2020). Though there is no specific antiviral treatment available, people infected with the SARS-CoV-2' receive medical and supportive care to relieve their symptoms. A worldwide race is underway to create and mass-produce a successful immunization to counter the COVID-19. WHO and various governmental organizations have cautioned that way of life cannot return to typical until their populaces have built up antibodies to fight off the infection. Quickened clinical trials are as of now underway, but antibody advancement regularly takes a longer time.

Qatar reported the first case of COVID-19 on 29 th February 2020. As of $25^{\text {th }}$ July 2020, Qatar's case fatality rate is at $0.2 \%$ with only 164 deaths $(\mathrm{MOPH}$, Qatar, 2020). Physiotherapists are an integral part of the multidisciplinary team (MDT) focusing on the early mobilization of critically ill patients along with their vital role in pulmonary hygiene. Critical care physiotherapists in Qatar are additionally skilled in the rehabilitation of patients under ECMO care. Supportive studies are emerging to reinforce the beneficial effect of critical care physiotherapy in the management and rehabilitation of patients with COVID-19 along with its proven role to prevent or delay ICU acquired weakness, delirium and other complications arise from immobility induced from prolonged ventilation, sedation and use of neuromuscular blocking agents (Ngeh N E et al., 2020; Brugliera L et al., 2020; Righetti RF et al., 2020). An early rehabilitation can limit the immobility associated complications thereby promoting rapid functional recovery (Herridge $\mathrm{M}$ et al., 2011; Righetti RF et al., 2020).

Aim/objectives of the best practice
recommendation

- To provide evidence-based clinical recommendations to physiotherapists working in acute and critical care facilities for the management of suspected and/or confirmed COVID-19 patients.

- To safeguard and protect the physiotherapists working with such groups of patients.

\section{Scope}

This document focuses on the adult acute care hospital setting including critical care and ECMO care. This document has been prepared to provide information to physiotherapists and acute healthcare facilities about the potential role of physiotherapy in the management of acutely-hospital-admitted patients with confirmed or suspected COVID-19. While these recommendations were developed, the transmissibility of COVID-19, the principle of limited manpower exposure, and the level of care to be provided by physiotherapists were considered.

This recommendation outlines:

- Physiotherapy referral, screening and management considerations,

- Physiotherapy management categories and

- Physiotherapy best practice recommendations based on available evidences and the scope of practice of Qatar.

\section{Guideline Development Process}

This guideline is the result of a combination of systematic evidence search, subsequent critical evaluation of retrieved evidence and consensus process. An expert panel of physiotherapists with extensive experience in the field of critical care practice was created. As an initial step a systematic search on the available evidences on COVID-19 and critical care physiotherapy was conducted. Existing guidelines, recommendations, protocols, consensus statements and relevant publications were identified and distributed among the panel members mirroring the process followed in Vitacca et al's study (2020a).

The available evidences were critically appraised. Multiple virtual meetings were conducted to draft the skeleton of this recommendation. The panel members 
were divided into groups to work on specific areas, and later following a series of presentations, discussions and modifications, the final draft was created. The major aim of the guideline, 'to recommend the optimal physiotherapy care for the suspected and confirmed COVID-19 cases admitted in an acute-critical care facility' was cross checked at different time intervals to ensure that the recommendations are reflecting the aim. A final best practice recommendation was tailored ensuring the relevance, practicability and flexibility to suit various healthcare systems' functionality. Multiple feedback rounds were used before the final revision. When evidence was minimal, the consensus was developed for mandatory recommendations. Consensus was defined and ensured as $100 \%$ agreement among the panel members and the reviewers.

\section{A. Acute Care Physiotherapy COVID-19 Patient Management Algorithm (COVID-19-PTM Algorithm)}

The COVID-PTM algorithm outlines the physiotherapy management for COVID-19 patients in the acute hospital setting based on the acuity, severity and mobility of the patients. It classifies the suspected and confirmed COVID-19 patients into various categories, recommending appropriate care and optimizing workforce planning and preparation. The algorithm can be used as a screening tool for determining the requirement of physiotherapy, recommendations of optimal physiotherapy management and infection control procedures. The algorithm is based on international consensus and designed to minimize the staff exposure and risk (thus reducing the probability of spreading the infection and preventing the risk of high numbers of staff being unavailable for duty) while maintaining adequate levels of service to minimize the adverse effects of immobility (Figure 1).

Target audience: Physiotherapists, components of multidisciplinary team (MDT), and other relevant stakeholders in the critical-acute care setting caring for adult patients with suspected and/or confirmed COVID-19.

Target setting: All COVID-19 hospitals or any critical and acute care facility handling adult patients with suspected and/or confirmed COVID-19.

\section{B. Best Practice Recommendations}

Section 1: Physiotherapy referral, screening and management considerations

\subsection{Physiotherapy referral}

1.1.1 Follow the guidelines and policies of your institution regarding physiotherapy referral for patients admitted in an acute care setting seeking or referred for physiotherapy

1.2 Screening and categorization of referred patients

1.2.1 Physiotherapist should carry out a thorough case evaluation remotely through the electronic documentation system.

1.2.2 Patients should be categorized according to the 'Acute Care Physiotherapy COVID-19 Patient Management Algorithm (COVID-19PTM algorithm)' (Figure 1)

1.2.3 Patients admitted 'without' any COVID-19 associated symptoms (Eg:- Fever, cough, malaise, Shortness of breath (SOB), sore throat, headache, loss of taste/smell, conjunctivitis) should follow routine physiotherapy care adhering to standard infection control precautions.

1.2.4 Patients admitted 'without' any COVID-19 associated symptoms, but with suspicion of COVID-19 due to direct contact with COVID-19 patients (including quarantine) should be considered as 'suspected COVID-19' as per the COVID-PTM algorithm.

1.2.5 Patients admitted with any other causes (Cardiac, trauma, orthopedic or neurological) and with COVID-19 associated symptoms must be categorized as 'suspected COVID 19' as per the COVID-19-PTM algorithm.

1.2.6 All the patients admitted 'with' COVID-19 associated symptoms must be categorized as 
'suspected COVID-19' as per the COVID-19-PTM algorithm.

\subsection{Physiotherapy management considerations}

1.3.1 All suspected or confirmed cases should be reported to the managers through the established channels and should be recorded in a COVID-19 physiotherapy database, with all the mandatory status updates according to the institutional policies and departmental procedures.

1.3.2 All suspicious cases of COVID-19 should be considered as COVID 19 patients until confirmed by approved test protocols and they need to be followed with all 2.2 precautionary measures as per the institution's infection control policies.

1.3.3 All the COVID 19 positive cases should be 'delegated to' the 'COVID-19 physiotherapy team' to continue the care. This will help to minimize / prevent infection spread while offering optimal care.

1.3.4 The management of the patient should follow the COVID-PTM algorithm (Figure 1)

\section{Section 2: Physiotherapy management categories}

\subsection{Category A: Intubated, sedated and paralyzed} patients

2.1.1 Includes patients who are critically ill, intubated, sedated, paralyzed and maybe in prone position or on ECMO support or both.

2.1.2 The main goals of physiotherapy intervention at this stage are (Juultje, S. et al., 2015; Vitacca M et al., 2020b)

2.1.2.1 To minimize the complications of the prolonged recumbent position

2.1.2.2 To facilitate oxygenation

2.1.3 Physiotherapy management may include passive range of motion ( $\mathrm{ROM}$ ) exercises and therapeutic positioning. (Vitacca $\mathrm{M}$ et al., 2020b)

2.1.4 Physiotherapists must minimize/limit the exposure to these patients judiciously to prevent unnecessary usage of personal protective equipment (PPE) and risk of cross-contamination.

2.1.5 Collaborate with MDT for therapeutic positioning and joint protection techniques to minimize or limit the exposure.

2.1.6 Decision of rendering physiotherapy management should depend upon the rehabilitation potential of the patient, and considering risk vs. benefits based on the disease status.

2.1.7 Regular communication with MDT regarding patient status and ongoing care including physiotherapy.

2.2 Category B: Intubated, minimally sedated/ on sedation vacation patients

2.2.1 Includes patients who are ill, intubated, sedated to a minimum or on a sedation vacation, maybe in prone position or on ECMO support but 'not-paralyzed.'

2.2.2 Develop an individualized treatment plan based on the patient's conscious level, level of cooperation, hemodynamic status and critical care supports.

2.2.3 The main goals of physiotherapy intervention at this stage are

2.2.3.1 To minimize the complications of the prolonged recumbent position (Lopez $\mathrm{M}$ et al., 2020)

2.2.3.2 To facilitate oxygenation

2.2.3.3 To facilitate early mobilization (Ngeh, N. et al., 2020)

2.2.4 Physiotherapy management may include therapeutic positioning, ROM exercises and early progressive mobilization.(Vitacca $\mathrm{M}$ et al., 2020b)

2.2.5 Limit or minimize the aerosol-generating procedures, and physiotherapists should weigh up the risk versus benefit for initiating these procedures. If necessary, extreme precautions should be followed while performing these procedures. (Refer Section 3.1 for recommendations).(Vitacca $\mathrm{M}$ et al., 2020b; Vitacca M et al., 2020a) 


\subsection{Category C: Non- intubated patients}

2.3.1 These patients are divided into 3 categories depending on their ventilatory supports, conscious level, and functional independence into category C.1, C.2 and C.3.

2.3.2 The main goals of physiotherapy interventions are

2.3.2.1 To reduce the work of breathing

2.3.2.2 To improve lung capacity

2.3.2.3 To facilitate oxygenation

2.3.2.4 To maximize functional independence (Ngeh NE et al., 2020)

2.3.3 Category C.1: Minimally conscious and bedridden patients

2.3.3.1 Includes patients who are ill, non-intubated, non-sedated, minimally conscious, bedridden patients, with probably some sort of non-invasive ventilatory support.

2.3.3.2 Physiotherapy interventions may include passive ROM exercises, stretching, therapeutic positioning, verticalization or out of bed activities as indicated/tolerated. (Vitacca $\mathrm{M}$ et al., 2020b)

2.3.3.3 Physiotherapy treatment progression and frequency depends upon the patient's rehabilitation potential, overall health and disease status.

2.3.3.4 Regular follow-up with the MDT on patient's adherence to exercise program and therapeutic positioning.

2.3.4 Category C.2: Conscious, active and dependent patients

2.3.4.1 Includes patients who are non-intubated, non-sedated, conscious patients who need assistance with their ADL, with or without a non-invasive ventilatory support.

2.3.4.2 Physiotherapy interventions may include respiratory physiotherapy, ROM exercises, stretching, progressive strengthening exercises, progressive mobilization, cardiopulmonary rehabilitation, exercises to improve coordination and balance etc. (Vitacca M et al., 2020b)

2.3.4.3 Risk and benefit should be considered before providing respiratory physiotherapy techniques. Airway clearance techniques should be continued only for hyper secretive patients. Strict airborne infection practices should be followed during the respiratory physiotherapy session. (Vitacca M et al., 2020a)

2.3.4.4 Develop an individualized treatment plan based on patient's oxygen dependency, neuromuscular, cardiopulmonary abilities and functional capacity.

2.3.4.5 Mobilization should be considered as an aerosol-generating procedure as it can lead to coughing or expectoration of secretions. Utmost caution should be followed while performing these techniques (Refer section 3.2 for recommendations).

2.3.4.6 Dedicated mobility aids to be kept in the isolation area for the use of COVID 19 patients. (CDC, 2020; Cieloszczyk et al., 2020)

2.3.4.7 Ensure appropriate resources (manpower as well as equipment) before initiating mobilization or therapeutic programs.

2.3.4.8 Mobility aids that are required should be tagged and left in the patient's room or cleaned and disinfected thoroughly if to be reused for other patients. (CDC, 2020 )

2.3.4.9 Avoid the use of specialized and large equipments as much as possible or disinfect thoroughly if used for COVID 19 patients. (CDC, 2020 ) 
2.3.4.10 Regular follow-up with the MDT on patient's adherence to exercise program.

2.3.4.11 Strict obedience to the infection control procedures and precautions while performing the mobilization or therapeutic exercises. (Cieloszczyk et al., 2020; CDC, 2020; Thomas P et al., 2020)

2.3.5 Category C.3: Conscious, active \& independent patients

2.3.5.1 Includes patients who are non-intubated, non-sedated, conscious and who are independent, modified independent or supervised with their ADLs, with or without a non-invasive ventilatory support.

2.3.5.2 Physiotherapy interventions may include supervised or semi supervised exercise programs such as ROM exercises, progressive ambulation, breathing control and other therapeutic exercises as indicated.

2.3.5.3 Risk and benefit should be considered before providing respiratory physiotherapy techniques. Airway clearance techniques should be continued only for hyper secretive patients. Strict airborne infection practices should be followed during the respiratory physiotherapy session. (Vitacca M et al., 2020a)

2.3.5.4 Develop an individualized exercise program based on the patient's endurance and disease status. Aerobic low load exercise based on subjective symptoms with low intensity are recommended. (Vitacca $\mathrm{M}$ et al., 2020a)

2.3.5.5 Limit or minimize the exposure by educating the patient on a safe exercise program and encourage continuing the exercise program independently.

2.3.5.6 Regular follow-up with the MDT to ensure patient's adherence to exercise program and modifications as per the patient's activity tolerance.

\subsubsection{Algorithm based manpower utilization}

2.3.6.1. Because of a sudden surge in the number of cases, the introduction of COVID-19 and field hospitals, and the expansion of critical care beds, there is a massive shortage of healthcare professionals across the hospitals necessitating careful manpower redistribution. The COVID-PTM algorithm (Figure 1) can be used effectively to redistribute the manpower while maintaining optimal care. Patients in the category B \& C require more active interventions than category A, where the therapy is more passive. In the category $\mathrm{C}$ the subcategory C.2 requires more active and one to one care than C.1 \& C.3 subcategories. The C.3 subcategory requires more supervised exercise programs, mobility care and education as this group comprises conscious, active and independent patients. Table 1 gives a model of staff redistribution based on the full-time equivalent (FTE) calculation. Here, as a model one FTE is calculated as nine COVID beds with dedicated physiotherapy care. This calculation will be variable based on the FTE definition of various organizations and specialties. The table gives us a calculation of a gain of 1.5 FTE (total FTE 2-FTE 1) when the care delivery was readjusted based on the COVID-PTM algorithm model compared to the normal (FTE 1).

Section 3: Therapeutic best practice 


\subsection{Recommendations on respiratory interventions}

3.1.1 Respiratory interventions like postural drainage, intense breathing exercises/techniques, and secretion clearance techniques are potentially aerosol-generating procedures and increase the work of breathing. (Simonelli et al., 2020; Vitacca M et al., 2020a)

3.1.2 Avoid/ minimize these interventions and physiotherapists should weigh up the risk versus benefit to initiate these interventions. (Simonelli et al., 2020; CDC, 2020; Vitacca M et al., 2020a)

3.1.3 Airborne precautions should be followed while performing respiratory interventions. (refer 3.4 recommendations)

3.1.4 The N95 respirator must fit the user's face snugly (i.e., create a seal) to minimize the number of particles that bypass the filter through gaps between the user's skin and the respirator seal. (Lazzeri M et al., 2020; Ferioli M et al., 2020)

3.1.5 Appropriate doffing and donning techniques should be followed for all personal protective equipment (PPE) as per the infection control policies. (CDC, 2020)

3.1.6 Physiotherapists should follow (if possible) posterior or lateral approach while mobilizing the patients and should position themselves $\geq$ $2 \mathrm{~m}$ from the patient (if possible) to be out of the "blast zone" or line of cough (Cieloszczyk et al., 2020; Simonelli et al., 2020)

3.1.7 Face mask/reservoir mask is preferable over the nasal cannula while mobilizing a patient (face mask with oxygen flow up to $5 \mathrm{~L} / \mathrm{min}$, a reservoir mask up to $10 \mathrm{~L} / \mathrm{min}$ of $\mathrm{O}_{2}$ or a Venturi mask up to $60 \%$ of $\mathrm{FiO}_{2}$ ) to minimize the dispersion of droplets, with a surgical mask covering the patient face over it. If this mask is soiled it should be discarded immediately as per the infection control policies and wear a new mask (Hui DS et al., 2006; Ferioli M et al., 2020).

3.1.8 If a nasal cannula is the only option, it should be well-positioned inside the nostrils and a surgical mask should be added over the cannula. The same rule is applicable if the patient uses HFNO therapy. (Lazzeri M et al., 2020; CDC, 2020; ANZICS, 2020)

3.1.9 If the patient is on noninvasive ventilation (CPAP/BIPAP), ensure that there is no air leak before starting any physiotherapy exercise session. Also, care should be taken to avoid the disconnection of the machine connection circuit. (Simonelli et al., 2020)

3.1.10 A NIV mask connected by T-tube to the circuit can be used for patients who are open-mouth breathers as a way to improve the oxygen saturation during the exercise session. (Lazzeri M et al., 2020)

3.1.11 Positioning to improve oxygenation: The patient can be better placed in a semi-sitting or sitting position while avoiding slumped sitting. Alternations of lateral decubitus, semi-prone or prone positions might be beneficial as far as the oxygenation is concerned (Ding et al., 2020). The positions should be passive to reduce muscular activity and to relax the accessory muscle to facilitate the ventilation/perfusion ratio. Cushions/pillows can be used to attain the optimum (passive) resting positions (Lazzeri M et al., 2020).

\subsection{Recommendations on mobilization interventions}

3.2.1 Mobilization should be considered as an aerosol-generating procedure as it leads to coughing or the expectoration of secretions. It also requires close contact with the patients by physiotherapist. Therefore, strict care should be taken when conducting these procedures with airborne precautions. (Refer 3.4.2 recommendations). 
3.2.2 While mobilizing mechanically ventilated patients, extreme care should be taken to maintain the ventilator circuit in place. (Cieloszczyk et al., 2020)

3.2.3 Non-mechanically ventilated patients should wear a mask during the physiotherapy sessions. (Cieloszczyk et al., 2020)

3.3. Procedures that should be used with extreme caution during the acute phase

3.3.1. When necessary, aerosol generating procedures should be carried out with extreme caution, since it is one of the COVID-19 transmission routes (CDC, 2020; ANZICS, 2020)

3.3.2. Table 2 outlines the various aerosol generating procedures related to physiotherapy (PT-AGPs) (Vitacca M et al., 2020a)

3.3.3. It is essential that physiotherapy procedures do not cause increased stress on the work of breathing, leading the patient to an increased risk of respiratory distress (Lazzeri $\mathrm{M}$ et al., 2020; Vitacca M et al., 2020a).

\section{Section 4: Extra Corporeal Membrane Oxygenation (ECMO)}

4.1. ECMO provides temporary mechanical support for the heart and lungs in patients with severe respiratory and or cardiovascular failure. There are 2 types of ECMO - Veno-Venous (VV) ECMO supporting primarily the lung function and Veno-Arterial (VA) ECMO supporting both heart and lungs. (Allen S et al., 2011; Peek GJ et al., 2009). 4.2. In critically ill patients with COVID-19, ECMO plays a potential role in the stabilization and their survival. (Loforte A et al., 2020; Jacobs JP et al., 2020)

4.3. Early physiotherapy in ECMO patients has been proven to be safe and feasible and to have a positive impact on outcomes. (Polastri M et al., 2016; Munshi L et al., 2017)

4.4. The majority of the COVID-19 patients who have severe acute respiratory compromise and persistent hypoxemia (ARDS) refractory to conventional management are indicated for the VV
ECMO. (Held N and Moss M, 2019; Loforte A et al., 2020; Jacobs JP et al., 2020)

4.5. The patients on ECMO may fall under category A, B, or C of the COVID-19-PTM Algorithm (figure 1) based on the critical supports they are receiving. The clinical decision making for the physiotherapy management of this group of patients will depend upon the category and mobilization guidelines for the ECMO patients. (Eden A et al., 2017)

4.6. There is growing evidence that early physiotherapy intervention for ECMO patients helps in early weaning from the ECMO and the mechanical ventilator, reducing the incidence of ICU acquired weakness, the length of hospital stay, and enhancing the lung function. (Ferreira et al., 2019; Ko et al., 2015: Polastri et al 2016)

4.7. Even though it is too early to search for evidence for the early mobilization of COVID-19 ECMO patients (Shekhar et al., 2020), in Qatar, any patient in the ECMO supports undergoes a standardized early mobilization protocol with varying infection control protocols as per the condition. The same is true in the COVID-19 cases too. The team has successfully implemented the progressive mobilization of the COVID-19 patients without any documented serious adverse events or status deterioration and with an average change in ICU mobility scale score of 2.5 out of 10 (range 0 to 7 ), where ' 0 ' is bedridden and 10 is independence in walking. (Hodgson et al., 2014; Tipping et al., 2016)

5. Ongoing physiotherapy care:-Post-acute care physiotherapy is an important component of patient care post COVID -19 to address and manage the complications associated with the critical care stay and also the consequences of the disease itself. (Sheehy LM, 2020) These may include impaired pulmonary function; prolonged immobilization based physical deconditioning; ICU acquired weakness, cognitive impairments such as delirium and psychological issues including post-traumatic stress disorders (PTSD) (Needham et al, 2012). A comprehensive evaluation and formulation of an individualized, progressive treatment plan focusing 
on function, disability, and return to participation in society is essential to maximize the quality of life (QOL) of the patients. (Sheehy LM, 2020) Physiotherapy needs could even be amplified by underlying health conditions and decrements in health associated with aging.

\subsection{Impaired Pulmonary Function: Most} COVID-19-admitted patients have compromised pulmonary function that needs supplemental oxygen therapy. Pulmonary impairment can progress from mild illness to pneumonia, extreme pneumonia, ARDS and even death (WHO, 2020(c,d)). Pulmonary rehabilitation play and important role in facilitating recovery, improving lung function and enhancing community re integration by promoting mobility and health related quality of life (Yang LL et al., 2020; Vitacca $M$ et al., 2020a). The following programs are recommended during the initial inpatient phase (Lazzeri M et al., 2020; Thomas P et al., 2020; Middleton, 2020; Zhao H-M et al., 2020; Kho ME et al., 2020; Vitacca M et al., 2020a).

5.1.1. Therapeutic positioning: to maintain and improve oxygenation and optimal functioning of primary muscles of respiration

5.1.2. Respiratory therapy including bronchial hygiene

5.1.3. Early progressive mobilization

\subsection{ICU Acquired Weakness (ICUAW):}

ICUAW is an important growing clinical problem among patients admitted to the ICU characterized by an acute onset decrease in muscular strength and muscle mass, deconditioning, myopathies, and neuropathies. (Kress J \& Hall J, 2014; de Jonghe et al., 2009). The common risk factors of ICUAW includes sepsis, systemic inflammation, steroid therapy, immobilization, and neuromuscular blocking agents. (Yang T et al., 2018) Thus COVID-19 patients are at high risk of developing ICUAW The best practice recommendations which have been shown to influence ICUAW are
5.2.1. Early passive mobilization (Hanekom S et al., 2011; Jacob P et al., 2020)

5.2.2. Electrical Stimulation (Wageck et al., 2014; Maffiuletti NA, 2010)

5.2.3. Early active mobilization (Hodgson et al., 2016; Kayambu G, Boots R and Paratz J, 2015; Jacob P et al., 2020)

5.2.4. Cycle ergometry (Hodgson CL et al., 2013)

\subsection{Physical deconditioning: Physical} deconditioning during the acute hospital stay can be prevented or delayed by the judicious application of exercises, early mobilization, and progressive strengthening practices as early as possible (Candan SA et al., 2020; Jacob P et al., 2020; Lazzeri M et al., 2020; Thomas P et al., 2020). Following COVID-19, patients will be physically deconditioned due to prolonged immobility, impaired lung function, and ICUAW. (PAHO, 2020)

\subsection{Post-traumatic Stress Disorders} (PTSD): PTSD is an anxiety disorder characterized by multiple psychological symptoms such as re-experiencing the trauma through persistent distressing memories of the incident, emotional numbness and avoidance of trauma-reminiscent circumstances, hyper-arousal issues such as difficulties in sleeping and concentrating, anxiety and irritability (Ehlers A and Clark DM, 2000). Due to its high infectivity, social and travel restrictions, death rate or threat of death, fear of survival and fear of illness, the occurrence of PTSD seems to be high during this pandemic period. (Liu N et al., 2020; Lopez M et al., 2020). Studies have proved that early mobilization, exercise, relaxation training, teaching stress and anxiety coping strategies, social and community integration and participation have a positive impact on the development of PTSD and related issues (Lazzeri M et al., 2020, Thomas P et al., 2020, 
Jacob P et al., 2020; Kho ME et al., 2020; Jackson JC et al., 2014).

\subsection{Role of Physiotherapy in Delirium} management: Delirium is defined as a disturbance of consciousness characterized by decreased knowledge of the environment, disorganized thought and impaired cognitive function (ASA, 2013). Delirium can lead to increased length of stay in hospital and increased complications. (Ahmed S et al., 2014). COVID-19 patients are at high risk of developing delirium due to sepsis, advanced age, sedative medications, immobility, social isolation, and ICUAW. Uncertainty about the pandemic situation, lack of empathy secondary to increased healthcare professional workload is also a high risk factor for development of delirium among COVID-19 patients. (Kotfis et al., 2020; Lopez M et al., 2020). Early mobilization, relaxation training, sensory stimulation, teaching stress and anxiety coping strategies are helpful in preventing or reducing the incidence of delirium (Lazzeri M et al., 2020, Jacob P et al., 2020; Kho ME et al., 2020; Jackson JC et al., 2014). The physiotherapist has a crucial role in the prevention and management of delirium and its associated complications (Lazzeri M et al., 2020; Middleton, 2020)

\section{Summary}

The current epidemiological situation is a huge challenge for all medical professionals. It is estimated today that approximately $15-20$ percent of patients with COVID-19 require hospitalization and 5-6 percent require intensive care for a longer duration (WHO, 2020(b)). As is often the case during this type of pandemic, medical staff members themselves are particularly exposed to infection and need to take adequate measures to counteract that. Physiotherapists are an integral part of the multidisciplinary team of acute hospital wards and ICUs. Early physiotherapy can limit the immobility associated complications thereby promoting rapid functional recovery and reduced length of stay. The intention of this best practice recommendation is to streamline and optimize the physiotherapy care delivery for critical $\&$ acute care COVID-19 patients, while at the same time stressing the need to uphold clearly established safety guidelines.

Author Contributions: Conceptualization: MS Ajimsha, Neeraj Gampawar, Praveen J Surendran, Prasobh Jacob.; Methodology, MS Ajimsha \& Praveen J Surendran; guideline committee coordinating: Prasobh Jacob; plagiarism \& Validation: Vasileios Karpouzis and Mohamed Haneef; Content analysis and referencing Muhamed Aleef \& Shameem Ali , Investigation, Reshma Praveen.; Resources, \& Data verification Eladel Bouguerra, \& Noora Almudahka Writing - Original Draft Preparation, MS Ajimsha \& Neeraj Gampawar Writing - Review \& Editing, MS Ajimsha,; Praveen J Surendran,; Prasobh Jacob,; Vasileios Karpouzis, Supervision: Noora Almudahka; Project Administration, MS Ajimsha \& Noora Almudahka

Funding: This research received no external funding

Conflicts of Interest: "The authors declare no conflict of interest."

\section{References:}

1. Ahmed S, Leurent B, Sampson EL. Risk factors for incident delirium among older people in acute hospital medical units: a systematic review and meta-analysis. Age Ageing. 2014;43(3):326-333. doi:10.1093/ageing/afu022

2. Allen S, Holena D, McCunn M, Kohl B, Sarani B. A review of the fundamental principles and evidence base in the use of extracorporeal membrane oxygenation (ECMO) in critically ill adult patients. J Intensive Care Med. 2011;26(1):13-26.

3. Australian and New Zealand Intensive Care Society. ANZICS COVID-19 Guidelines, 2020. Melbourne: ANZICS; 2020.

4. Brugliera L, Spina A, Castellazzi P, et al. Rehabilitation of COVID-19 patients. $J$ 
Rehabil Med. 2020;52(4):jrm00046.

Published $2020 \quad$ Apr 15.
doi:10.2340/16501977-2678

5. Candan, S. A., Elibol, N. and Abdullahi, A. (2020) 'Consideration of prevention and management of long-term consequences of post-acute respiratory distress syndrome in patients with COVID-19', Physiotherapy Theory and Practice. Taylor \& Francis, 36(6), pp. 663-668. doi: $\underline{10.1080 / 09593985.2020 .1766181 .}$.

6. CDC (2020) Coronavirus Disease 2019 (COVID-19), Centers for Disease Control and Prevention. Available at: https://www.cdc.gov/coronavirus/2019-ncov /hcp/infection-control-recommendations.htm 1 (Accessed: 25 July 2020).

7. Chen J, Fan H, Zhang L, et al. Retrospective analysis of clinical features in 101 death cases with COVID-19. medRxiv 2020; Published Ahead of Print:2020.03.09.20033068. doi: 10.1101/2020.03.09.20033068 (a)

8. Cieloszczyk A, Lewko A, Śliwka A, Włoch T, Pyszora A, 2020. Recommendations of the Polish Chamber of Physiotherapists for physiotherapy of adult patients with COVID-19. Krajowa Izba Fizjoterapeutów. URL

https://kif.info.pl/recommendations-of-the-p olish-chamber-of-physiotherapists-for-physi otherapy-of-adult-patients-with-covid-19/ (accessed 7.25.20).

9. de Jonghe B, Lacherade JC, Sharshar T, Outin H 2009 Intensive care unit-acquired weakness: Risk factors and prevention. Critical Care Medicine 37: 309-315. doi:10.1097/CCM.0b013e3181b6e64c.

10. del Rio C, Malani P. 2019 Novel Coronavirus-Important Information for Clinicians. JAMA. 2020;323(11):1039.

11. Dijkstra-Kersten SMA, Kok L, Kerckhoffs $\mathrm{MC}$, et al. Neuropsychiatric outcome in subgroups of Intensive Care Unit survivors: implications for after-care. $J$ Crit Care 2020;55:171-76 (available at: https://doi.org/10.1016/j.jcrc.2019.11.006, accessed 8 April 2020).

12. Ding L, Wang L, Ma W, He H. Efficacy and safety of early prone positioning combined with HFNC or NIV in moderate to severe ARDS: a multi-center prospective cohort study. Critical care. 2020 Dec 1;24(1):28

13. Eden A, Purkiss C, Cork G, et al. In-patient physiotherapy for adults on veno-venous extracorporeal membrane oxygenation United Kingdom ECMO Physiotherapy Network: A consensus agreement for best practice. $J$ Intensive Care Soc. 2017;18(3):212-220.

14. Ehlers A, Clark DM. A cognitive model of posttraumatic stress disorder. Behaviour research and therapy. $2000 \mathrm{Apr}$ 1;38(4):319-45.

15. Ferioli M, Cisternino C, Leo V, Pisani L, Palange P, Nava S. Protecting healthcare workers from SARS-CoV-2 infection: practical indications. Eur Respir Rev. 2020;29(155):200068.

16. Ferreira D.Ddc, F., Maz, M., Fe, M., Rdm, P. and A, K. 2019. Safety and potential benefits of physical therapy in adult patients on extracorporeal membrane oxygenation support: a systematic review. Revista Brasileira de Terapia Intensiva. 31(2), pp.227-239.

17. Guan W, Ni Z, Hu Y, Liang W, Ou C, He J et al. Clinical Characteristics of Coronavirus Disease 2019 in China. New England Journal of Medicine. 2020.

18. Hanekom S, Gosselink R, Dean E, van Aswegen H, Roos R, Ambrosino N, Louw Q 2011 The development of a clinical management algorithm for early physical activity and mobilization of critically ill patients: Synthesis of evidence andexpert 
opinion and its translation into practice. Clinical Rehabilitation 25: 771-787. doi:10.1177/0269215510397677.

19. Held N, Moss M. Optimizing post-intensive care unit rehabillitation. Turkish Thoracic Journal 2019;20(2):147-52.

20. Herridge M, Tansey C, Matté A, Tomlinson G, Diaz-Granados N, Cooper A et al. Functional Disability 5 Years after Acute Respiratory Distress Syndrome. New England Journal of Medicine. 2011;364(14):1293-1304.

21. Hodgson CL, Bailey M, Bellomo R, Berney S, Buhr H, Denehy L, et al. A binational multicenter pilot feasibility randomized controlled trial of early goal-directed mobilization in the ICU. Crit Care Med. 2016;44:1145-1152

22. Hodgson CL, Berney S, Harrold M, Saxena M, Bellomo R. Clinical review: Early patient mobilization in the ICU. Crit Care. 2013;17:207.

23. Hodgson, CL., Needham, D., Haines, K., Bailey, M., Ward, A., Harrold, M., Young, P., Zanni, J., Buhr, H., Higgins, A., Presneill, J. and Berney, S. 2014. Feasibility and inter-rater reliability of the ICU Mobility Scale. Heart \& Lung: The Journal of Critical Care. 43(1), pp.19-24.

24. Hui DS, I Azhar E, Madani T, Ntoumi F, Kock $\mathrm{R}$, Dar $\mathrm{O}$ et al. The continuing 2019-nCoV epidemic threat of novel coronaviruses to global health — The latest 2019 novel coronavirus outbreak in Wuhan, China. International Journal of Infectious Diseases. 2020;91: 264-266.

25. Hui DS, Ip M, Tang JW, Wong AL, Chan MT, Hall SD, Chan PK, Sung JJ. Airflows around oxygen masks: A potential source of infection. Chest. 2006 Sep 1;130(3):822-6

26. Jackson JC, Pandharipande PP, Girard TD, Brummel NE, Thompson JL, Hughes CG, et al. Depression, post-traumatic stress disorder, and functional disability in survivors of critical illness in the BRAIN-ICU study: a longitudinal cohort study. The Lancet Respiratory Medicine. 2014;2(5):369-79.

27. Jacob, P., Surendran, P., E M, M., Papasavvas, T., Praveen, R., Swaminathan, N. and Milligan, F., 2020. Early Mobilization of Patients Receiving Vasoactive Drugs in Critical Care Units. Journal of Acute Care Physical Therapy, Publish Ahead of Print.

28. Jacobs JP, Stammers AH, St Louis J, et al. Extracorporeal Membrane Oxygenation in the Treatment of Severe Pulmonary and Cardiac Compromise in Coronavirus Disease 2019: Experience with 32 Patients. ASAIO J. 2020;66(7):722-730. doi:10.1097/MAT.0000000000001185

29. Juultje Sommers Physiotherapy in the intensive care unit: an evidence-based, expert driven, practical statement and rehabilitation recommendations 2015

30. Kayambu G, Boots R, Paratz J. Early physical rehabilitation in intensive care patients with sepsis syndromes: a pilot randomised controlled trial. Intensive Care Med. 2015;41:865-874.

31. Kho ME, Brooks D, Namasivayam-MacDonald A, Sangrar R, Vrkljan B. Rehabilitation for Patients with COVID-19 Guidance for Occupational Therapists, Physical Therapists, Speech-Language Pathologists, and Assistants [Internet]. 2020 [cited 2020 Apr 16]. Available from: https://srs-mcmaster.ca/wp-content/uploads/ 2020/04/Rehabilitation-for-Patients-with-C OVID-19-Apr-08-2020.pdf

32. Ko, Y., Cho, Y.H., Park, Y.H., Lee, H., Suh, G.Y., Yang, J.H., Park, C.-M., Jeon, K. and Chung, C.R. 2015. Feasibility and Safety of Early Physical Therapy and Active 
Mobilization for Patients on Extracorporeal Membrane Oxygenation. ASAIO journal (American Society for Artificial Internal Organs: 1992). 61(5), pp.564-568.

33. Kotfis, K., Williams Roberson, S., Wilson, J.E., Dabrowski, W., Pun, B.T. and Ely, E.W. 2020. COVID-19: ICU delirium management during SARS-CoV-2 pandemic. Critical Care. 24(1), p.176.

34. Kress J, Hall J. ICU-Acquired Weakness and Recovery from Critical Illness. New England Journal of Medicine. 2014;370(17):1626-1635.

35. Lazzeri M, Lanza A, Bellini R, Bellofiore A, Cecchetto S, Colombo A, D'Abrosca F, Del Monaco C, Gaudellio G, Paneroni M, Privitera E. Respiratory physiotherapy in patients with COVID-19 infection in acute setting: a Position Paper of the Italian Association of Respiratory Physiotherapists (ARIR). Monaldi Archives for Chest Disease. 2020 Mar 26;90(1).

36. Liu N, Zhang F, Wei C, et al. Prevalence and predictors of PTSS during COVID-19 outbreak in China hardest-hit areas: Gender differences matter. Psychiatry Res. 2020;287:112921.

doi:10.1016/j.psychres.2020.112921

37. Loforte A, Dal Checco E, Gliozzi G, et al. Veno-venous Extracorporeal Membrane Oxygenation Support in COVID-19 Respiratory Distress Syndrome: Initial Experience. ASAIO J. 2020;66(7):734-738. doi:10.1097/MAT.0000000000001198

38. Lopez M, Bell K, Annaswamy T, Juengst S, Ifejika N. COVID-19 Guide for the Rehabilitation Clinician: A Review of Nonpulmonary Manifestations and Complications. Am J Phys Med Rehabil. 2020;99(8):669-673. doi:10.1097/PHM.0000000000001479

39. Maffiuletti NA. Physiological and methodological considerations for the use of neuromuscular electrical stimulation. Eur J Appl Physiol. 2010;110:223-234.

40. Middleton K. Rehabilitation is key to recovery - during and after Covid-19 [Internet]. The Chartered Society of Physiotherapy. 21/0402020 [cited 2020 Apr 22]. Available from: https://www.csp.org.uk/blog/2020/04/rehabi litation-key-recovery-during-after-covid-19

41. Mikkelsen ME, Shull WH, Biester RC, et al. Cognitive, mood and quality of life impairments in a select population of ARDS survivors. Respirology 2009;14(1):76- 82. doi: 10.1111/j.1440-1843.2008.01419.

42. Ministry of Public Health [Internet]. Moph.gov.qa. 2020 [cited 12 April 2020]. Available from: https://www.moph.gov.qa/english

43. Munshi L, Kobayashi T, DeBacker J, et al. Intensive Care Physiotherapy during Extracorporeal Membrane Oxygenation for Acute Respiratory Distress Syndrome. Ann Am Thorac Soc. 2017;14(2):246-253. doi:10.1513/AnnalsATS.201606-484OC

44. Needham DM, Davidson J, Cohen H, et al. Improving long-term outcomes after discharge from intensive care unit: report from a stakeholders' conference, Crit Care Med 2012; 40(2): 502-09.

45. New coronavirus stable for hours on surfaces [Internet]. National Institutes of Health (NIH). 2020 [cited 12 April 2020]. Available from: https://www.nih.gov/news-events/news-rele ases/new-coronavirus-stable-hours-surfaces

46. Ngeh, N. E., Chigbo, N. N., Whitehouse, Z., Mukaruzima, L., Sichela, J.Z., Musago, K.I., Kitur, R. K., Shirima, R. M., Asouzu, N., Ekwueme, A., Rockson, A. A., Soliloye, O., Fanfon, T., Agoriwo, M. W., Anekwu, E.M., John, D. O., Mtsetfwa, L.V., Douryang, M., Busuulwa, A.,Nakawunde, 
C., Cockburn, L. Rehabilitation of patients with COVID-19 in African Settings: Guidance for Community Based Rehabilitation Workers, Physiotherapists, Occupational Therapists, Speech and Language Therapists, and Assistants. May 24, 2020. Available from https://www.wcptafrica.org/ and https://afri-can.org/

47. Parry SM, El-Ansary D, Cartwright MS, et al. Ultrasonography in the intensive care setting can be used to detect changes in the quality and quantity of muscle and is related to muscle strength and function. J Crit Care 2015;30(5):1151.e9-51. e14. doi: 10.1016/j.jcrc.2015.05.024

48. Peek GJ, Mugford M, Tiruvoipati R, Wilson A, Allen E, Thalanany MM, Hibbert CL, Truesdale A, Clemens F, Cooper N, Firmin RK, Elbourne D; CESAR trial collaboration. Efficacy and economic assessment of conventional ventilatory support versus extracorporeal membrane oxygenation for severe adult respiratory failure (CESAR): a multicenter randomised controlled trial. Lancet. 2009;374(9698):1351-63. Erratum inLancet. 2009;374(9698):1330.

49. Polastri M, Loforte A, Dell'Amore A, Nava

S. Physiotherapy for Patients on Awake Extracorporeal Membrane Oxygenation: A Systematic Review. Physiother Res Int. 2016;21(4):203-209. doi:10.1002/pri.1644

50. Rehabilitation considerations during the COVID-19 outbreak - PAHO/WHO | Pan American Health Organization (no date). Available at: http://www.paho.org/en/documents/rehabilit ation-considerations-during-covid-19-outbre ak (Accessed: 25 July 2020).

51. Righetti RF, Onoue MA, Politi FVA, et al. Physiotherapy Care of Patients with Coronavirus Disease 2019 (COVID-19) - A Brazilian Experience. Clinics (Sao Paulo). 2020;75:e2017. Published 2020 Jun 22. doi:10.6061/clinics/2020/e2017

52. Sheehy LM. Considerations for Postacute Rehabilitation for Survivors of COVID-19. JMIR Public Health Surveill. 2020;6(2):e19462. Published 2020 May 8. doi:10.2196/19462

53. Shekar, K., Badulak, J., Peek, G., Boeken, U., Dalton, H.J., Arora, L., Zakhary, B., Ramanathan, K., Starr, J., Akkanti, B., Antonini, M.V., Ogino, M.T., Raman, L., Barret, N., Brodie, D., Combes, A., Lorusso, R., MacLaren, G., Müller, T., Paden, M. and Pellegrino, V. 2020. Extracorporeal Life Support Organization Coronavirus Disease 2019 Interim Guidelines: A Consensus Document from an International Group of Interdisciplinary Extracorporeal Membrane Oxygenation Providers. Asaio Journal.

54. Simonelli, C., Paneroni, M., Fokom, A.G., Saleri, M., Speltoni, I., Favero, I., Garofali, F., Scalvini, S. and Vitacca, M. 2020. How the COVID-19 infection tsunami revolutionized the work of respiratory physiotherapists: an experience from Northern Italy. Monaldi Archives for Chest Disease. 90(2).

55. Thomas P, Baldwin C, Bissett B, Boden I, Gosselink R, Granger CL, et al. Physiotherapy management for COVID-19 in the acute hospital setting: clinical practice recommendations. Journal of Physiotherapy [Internet]. 2020 Mar 30 [cited 2020 Apr 28]; Available from:

http://www.sciencedirect.com/science/article /pii/S183695532030028X

56. Tipping, C.J., Bailey, M.J., Bellomo, R., Berney, S., Buhr, H., Denehy, L., Harrold, M., Holland, A., Higgins, A.M., Iwashyna, T.J., Needham, D., Presneill, J., Saxena, M., Skinner, E.H., Webb, S., Young, P., Zanni, J. and Hodgson, C.L. 2016. The ICU Mobility Scale Has Construct and Predictive 
Validity and Is Responsive. A Multicenter Observational Study. Annals of the American Thoracic Society. 13(6), pp.887-893.

57. Vitacca M, Lazzeri M, Guffanti E, et al. Italian suggestions for pulmonary rehabilitation in COVID-19 patients recovering from acute respiratory failure: results of a Delphi process. Monaldi Arch Chest Dis. 2020;90(2):10.4081/monaldi.2020.1444. Published 2020 Jun 23 (a)

58. Vitacca M, Carone M, Clini EM, et al. Joint Statement on the Role of Respiratory Rehabilitation in the COVID-19 Crisis: The Italian Position Paper. Respiration. 2020;99(6):493-499. doi:10.1159/000508399 (b)

59. Wageck B, Nunes GS, Silva FL, Damasceno MC, de Noronha M. Application and effects of neuromuscular electrical stimulation in critically ill patients: systematic review. Med Intensiva. 2014;38:444-454.

60. World Health Organization. (2020). Coronavirus disease 2019 (COVID-19): situation report, 46. World Health Organization. (a) https://apps.who.int/iris/handle/10665/33144 $\underline{3}$

61. WHO Director-General's opening remarks at the media briefing on COVID-19 - 11 March 2020 [Internet]. Who.int. 2020 [cited 12 April 2020]. Available from: https://www.who.int/dg/speeches/detail/who -director-general-s-opening-remarks-at-themedia-briefing-on-covid-19---11-march-202 $\underline{0}$ (b)
62. World Health Organization, 2020. Clinical management of COVID-19: interim guidance, 27 May $2020 \quad$ (No. WHO/2019-nCoV/clinical/2020.5). World Health Organization. (c)

63. World Health Organization. Clinical Management of severe acute respiratory infection when novel coronavirus (2019-nCoV) infection is suspected: Interim Guidance, 2020. p. WHO Reference number WHO/2019-nCoV/clinical/2020.4. (d)

64. Yang LL, Yang T. Pulmonary Rehabilitation for Patients with Coronavirus Disease 2019 (COVID-19) [published online ahead of print, 2020 May 14]. Chronic Dis Transl Med. 2020;6(2):79-86. doi:10.1016/j.cdtm.2020.05.002

65. Yang, T., Li, Z., Jiang, L., Wang, Y. and Xi, X. 2018. Risk factors for intensive care unit-acquired weakness: A systematic review and meta-analysis. Acta Neurologica Scandinavica. 138(2), pp.104-114.

66. Zhao H-M, Xie Y-X, Wang C.

Recommendations for respiratory rehabilitation in adults with COVID-19. Chinese medical journal [Internet]. 2020 Apr; Available from: http://europepmc.org/abstract/MED/322510 $02 "$ http://europepmc.org/abstract/MED/322510 $\underline{02}$

67. Zhou F, Yu T, Du R, Fan G, Liu Y, Liu Z et al. Clinical course and risk factors for mortality of adult inpatients with COVID-19 in Wuhan, China: a retrospective cohort study. The lancet. 2020; 395 (10229): 1054-1062.1 
Figure 1

\section{Acute Care Physiotherapy COVID Patient Management Algorithm}

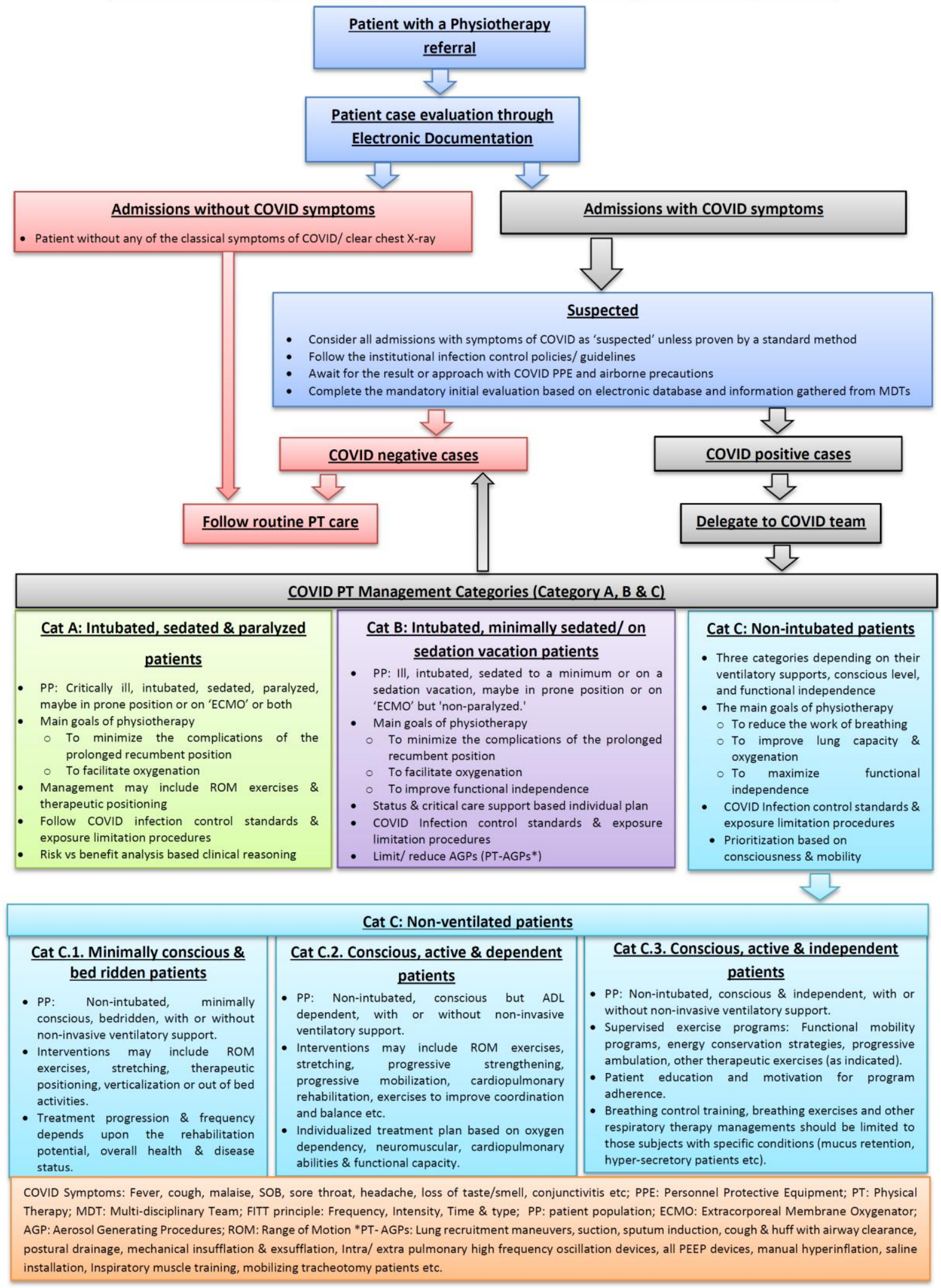


Table 1: Acute and critical care therapist-patient distribution model for COVID hospitals

\begin{tabular}{|l|l|l|l|}
\hline Category* & Patient population* & FTE1** & FTE2*** \\
\hline Category A & Intubated, sedated and paralyzed patients- Non ECMO & 1.0 & 0.5 \\
\hline Category B & Intubated, minimally sedated/ on sedation vacation patients & 1.0 & 1.0 \\
\hline Category C.1 & Minimally conscious and bedridden patients & 1.0 & 0.5 \\
\hline Category C.2 & Conscious, active and dependent patients & 1.0 & 1.0 \\
\hline Category C.3 & Conscious, active \& independent patients & 1.0 & 0.5 \\
\hline Category A, B or C & Patients on ECMO support & 1.0 & 1.0 \\
\hline Total & & $\mathbf{6}$ & $\mathbf{4 . 5}$ \\
\hline
\end{tabular}

* COVID-PTM algorithm model staff distribution (Considering one FTE as nine critical care patients / therapist); **FTE1:

Full time equivalent utilization non-COVID PTM algorithm model; ***FTE2: Full-time equivalent utilization COVID - PTM algorithm model

Table2: Physiotherapy aerosol generating procedures (PT-AGPs)

\begin{tabular}{|c|c|c|}
\hline Devices \& procedures & Breathing exercises & Mobilization \\
\hline $\begin{array}{l}\text { High flow nasal oxygen \& } \\
\text { Non-invasive ventilation (BiPAP, } \\
\text { CPAP) }\end{array}$ & $\begin{array}{l}\text { Deep \& diaphragmatic breathing } \\
\text { exercise }\end{array}$ & Turning and positioning \\
\hline Lung recruitment maneuvers & Pursed lips breathing & Postural Drainage \\
\hline Nebulization & $\begin{array}{l}\text { Chest Physiotherapy, vibration \& } \\
\text { shaking }\end{array}$ & $\begin{array}{l}\text { Mobilization of tracheostomy } \\
\text { patients }\end{array}$ \\
\hline $\begin{array}{l}\text { Open suctioning \& sputum } \\
\text { induction }\end{array}$ & Manual hyperinflation procedures & Bed mobility training \\
\hline Cuff inflation \& deflation & $\begin{array}{l}\text { Bronchial hygiene/lung re-expansion } \\
\text { techniques (Forced expiratory } \\
\text { techniques, coughing and huffing, } \\
\text { Tracheal stimulation etc. }\end{array}$ & Resistance training \\
\hline $\begin{array}{l}\text { Accidental disconnection of } \\
\text { ventilator circuits }\end{array}$ & Respiratory muscle training & Out of bed activities \\
\hline $\begin{array}{l}\text { Mechanical vibration, lifting and } \\
\text { standing devices }\end{array}$ & Saline installation & Transfer \& ADL training \\
\hline $\begin{array}{l}\text { Incentive spirometry, cough } \\
\text { machines etc }\end{array}$ & Stretching of the rib cage & Ambulation with assistance \\
\hline $\begin{array}{l}\text { High frequency oscillation } \\
\text { devises, all PEEP (Positive end } \\
\text { expiratory pressure) devises etc }\end{array}$ & Thoracic expansion & Endurance training \\
\hline
\end{tabular}

